\title{
Role of antibodies reactive to HspX in discriminating pulmonary tuberculosis contacts with high risk of developing active disease
}

\section{Dear Editor,}

Tuberculosis (TB) is a worldwide disease. It is estimated that one third of the world's population is infected with Mycobacterium tuberculosis (Mtb). ${ }^{1}$ Latent TB (Mtb infection) is detected by tuberculin skin test (TST). This test evaluates the cell-mediated immune response to purified protein derivative (PPD) obtained from culture of the Mtb complex bacteria. TB latently infected individual is characterized by Mtb infection without development of symptoms. TST is useful in screening for highly exposed individuals, such as contacts of TB patients and health care workers. However, the accuracy of this test depends on results interpretation. There are two possible interpretations for a positive TST: an old response that could be recalled in a booster phenomenon or a recent Mtb infection, which is called conversion. The interpretation of the TST result affects directly the physician's actions in order to avoid active TB development; it is well-known that a recent $\mathrm{TB}$ infected individual has 10\%-20\% more chances of developing active $\mathrm{TB}$ than individuals who had an old TB infection. It is also believed that individuals recently infected (those under TST positive conversion after exposure to an active TB patient) are at high risk of becoming ill, probably within two years after Mtb infection.

Prophylactic therapy with isoniazid was shown to be effective to avoid TB development among latently infected individuals. ${ }^{2}$ However, because one third of the world's population is infected with TB, this prophylaxis seems unlikely to be achieved. Therefore, the development of a quick test able to distinguish between TB latent individuals and those at high risk of developing active disease is urgent. We previously demonstrated that health care workers from a TB reference hospital who were recently infected (TST conversion) showed augmented and distinct antibody levels (IgM) against HspX Mtb. ${ }^{3}$ Along with this argument, we propose in this study to evaluate the application of an enzyme linked immunosorbent assay (ELISA) against HspX in the screening of TB recently infected individuals (TST converted) at high risk of developing active disease among contacts of pulmonary TB patients.

One hundred seventy-seven contacts from 45 pulmonary TB patients were submitted to TST. Upon patient's diagnosis, blood was harvested, after 4 weeks, 12 weeks, 6 months and 1 year, in order to evaluate the Mtb's infection (TST positive/conversion) and the dosages of IgM/IgG anti-HspX (ELISA), respectively. During the follow-up period, two contacts TST positive developed active TB, 8 converted their TST into positive and 6 boosted their TST. Table 1 shows the results of all contacts grouped by their socio-epidemiologic characteristics. ELISA for IgM-HspX did not reach satisfactory performance ( $\mathrm{ROC}$ area $=0.55$ ) and consequently all groups showed similar antibody levels. The role of antibodies in TB is discredited because Mtb's replication is intracellular ${ }^{4}$ and their use is restricted to diagnosis of active disease. However, it was recently shown that expression of $\mathrm{Fc} \gamma$ receptor can distinguish TB patients latently infected from non-infected individuals. ${ }^{5}$ We may speculate if the lowest anti-Hspx antibodies levels were due to a consumption of these molecules, which may play a role in Mtb infection in TST converted contacts. The other groups of contacts (TST negative, boosted), which presented higher levels of anti-HspX also did not develop active TB.

We concluded that IgM/IgG-HspX levels in latently infected individuals did not constitute a risk factor for the development of active tuberculosis. Therefore, it cannot be used to identify latent individuals who will develop active tuberculosis.
Authors

Michelle Cristina Guerreiro dos Reis Bruna Daniella de Souza Silva ${ }^{1}$

Eduardo Martins de Sousa $^{1}$

Ana Paula JunqueiraKipnis $^{2}$

${ }^{1} \mathrm{MSc}$, Instituto de Patologia Tropical e Saúde Pública, Universidade Federal de Goiás (UFG), GO, Brazil ${ }^{2} \mathrm{PhD}$, Instituto de Patologia Tropical e Saúde Pública, UFG, GO, Brazil

Submitted on: 05/21/2011 Approved on: 05/25/2011

Correspondence to: Ana Paula Junqueira-Kipnis Departamento de Microbiologia, Imunologia, Patologia e Parasitologia Instituto de Patologia Tropical e Saúde Pública Universidade Federal de Goiás

Rua 235 S/N

74605-050

Goiania, GO, Brazil Fax: +55 62 3209-6363 anapaula@iptsp.ufg.br

Financial Support: CNPq (project no. 575907/08-8), FUNAPE and FAPEG (project no. 001/2007).

We declare no conflict of interest.

(C2011 Elsevier Editora Ltda. All rights reserved. 
Table 1. Anti-HspX antibody levels of contacts of PTB patients according to socio-epidemiological characteristics

\begin{tabular}{|c|c|}
\hline & IgM-Hspx (O.D.) \\
\hline \multicolumn{2}{|l|}{ Gender } \\
\hline Female & $0.853 \pm 0.39(\mathrm{p}=0.53)$ \\
\hline Male & $0.805 \pm 0.36$ \\
\hline \multicolumn{2}{|l|}{ Age } \\
\hline$<16$ years & $0.895 \pm 0.36(p=0.30)$ \\
\hline 16 years & $0.816 \pm 0.37$ \\
\hline \multicolumn{2}{|l|}{ BCG } \\
\hline Yes & $0.805 \pm 0.35(p=0.25)$ \\
\hline No & $0.908 \pm 0.43$ \\
\hline \multicolumn{2}{|l|}{ Relatives } \\
\hline Yes & $0.849 \pm 0.39(\mathrm{p}=0.28)$ \\
\hline No & $0.761 \pm 0.31$ \\
\hline \multicolumn{2}{|l|}{ Same household } \\
\hline Yes & $0.828 \pm 0.38(\mathrm{p}=0.89)$ \\
\hline No & $0.823 \pm 0.33$ \\
\hline \multicolumn{2}{|c|}{ Contact frequency } \\
\hline Daily & $0.830 \pm 0.38(\mathrm{p}=0.96)$ \\
\hline Weekly & $0.798 \pm 0.27$ \\
\hline \multicolumn{2}{|l|}{ Kind of contact } \\
\hline Family & $0.849 \pm 0.38(\mathrm{p}=0.17)$ \\
\hline Institutional & $0.732 \pm 0.30$ \\
\hline \multicolumn{2}{|l|}{ Comorbidity } \\
\hline Present & $0.863 \pm 0.40(\mathrm{p}=0.48)$ \\
\hline Absent & $0.820 \pm 0.36$ \\
\hline
\end{tabular}

\section{REFERENCES}

1. WHO. Global Tuberculosis Control, a short update to 2009 report. WHO, 2009. [http://www.who.int/tb/publications/ global_report/2009/update/en/index.html].

2. Pineda NIS, Pereira SM, Matos ED, et al. Chemoprophylaxis in the prevention of tuberculosis. J Bras Pneumol. 2003; 342:268-72.

3. Rabahi MF, Junqueira-Kipnis AP, Reis MCG, et al. Humoral response to Hspx and GlcB to previous and recent infection by Mycobacterium tuberculosis. BMC Infect Dis. 2007; 31(7):148.

4. Casadevall A, Pirofski L. Antibody-mediated regulation of cellular immunity and the inflammatory response. Trends Immunol. 2003; 24(9):474-8.

5. Maertzdorf J, Repsilber D, Parida SK, et al. Human gene expression profiles of susceptibility and resistance in tuberculosis. Genes Immun. 2011; 12(1):15-22. 